\title{
MedienPädagogik
}

Zeitschrift für Theorie und Praxis der Medienbildung

\section{Mediatisierte Sozialisationsprozesse erforschen}

\section{Methodologische Implikationen}

\author{
Rudolf Kammerl, Claudia Lampert, Jane Müller, Marcel Rechlitz und \\ Katrin Potzel
}

\section{Zusammenfassung}

Mediatisierte Sozialisationsprozesse angemessen zu erfassen und zu analysieren, geht mit grossen Herausforderungen für die Forschung einher. Zum einen handelt es sich bei der Sozialisation um einen komplexen und langwierigen Prozess, an dem verschiedene Akteure beteiligt sind und der sich über verschiedene soziale Erfahrungsräume erstreckt. Zum anderen haben sich in der jüngeren Vergangenheit - neben anderen lebensweltlichen Voraussetzungen - die medialen Bedingungen, welche diesen Prozess rahmen, gravierend verändert und ausdifferenziert. Der vorliegende Beitrag erörtert, welche methodologischen Anforderungen die zunehmende Mediatisierung kindlicher Lebenswelten an die Sozialisationsforschung stellt. Er diskutiert diese vor dem Hintergrund eines oftmals einseitigen bzw. verkürzten Blickes der Forschung auf die Prozesse des Aufwachsens. Diesem stellt er den theoretischen Ansatz der kommunikativen Figurationen als Möglichkeit eines umfassenden Zugangs zu Sozialisation gegenüber. Implikationen und Herausforderungen des Ansatzes für die empirische Forschung werden anhand seiner konstituierenden Merkmale aufgezeigt. Dabei plädiert der Beitrag für den Einsatz qualitativer Längsschnittverfahren, um der Komplexität des Forschungsgegenstandes möglichst gerecht zu werden. 


\title{
Researching processes of mediatized socialisation. Methodological implications
}

\begin{abstract}
To adequately capture and analyze mediatized socialization processes poses significant challenges for research. On the one hand, socialization is a complex and lengthy process in which different actors are involved. This process spreads over different social spaces of experience. On the other hand, the media conditions that frame this process have changed and differentiated considerably in the recent past. This article discusses which methodological requirements the increasing mediatization of children's worlds poses to socialization research. It discusses these against the background of an often one-sided or shortened view of research on the processes of growing up. It contrasts this with the theoretical approach of communicative figurations as a possibility of comprehensive access to socialization. The implications and challenges of the approach to empirical research are demonstrated by its constituent features. The article advocates the use of qualitative longitudinal methods in order to take into account the complexity of the research subject.
\end{abstract}

\section{Zur Rolle der Medien in der Sozialisation}

Sozialisation ist der Prozess, bei dem sich Menschen mit ihren konstituierenden (biologischen) Grundlagen auf der einen Seite und den sie umgebenden sozialen sowie ökologischen Bedingungen auf der anderen Seite auseinandersetzen und dabei eine individuelle und soziale Persönlichkeit (Identität) entwickeln (Hurrelmann und Bauer 2015). Diese Entwicklung befähigt Individuen, sozial kompetent zu handeln und den mannigfaltigen, sich immer wieder ändernden Erwartungen ihrer sozialen Umwelt gerecht zu werden. Die Familienforschung charakterisiert die Bedingungen des Sozialisationsprozesses in der Kindheit insbesondere über die Dimensionen von Zugehörigkeit und Abgrenzung sowie von Kontrolle und Autonomie (Steinberg 2001). In verschiedenen Kontexten müssen sich Menschen verorten, indem sie sich zuordnen bzw. abgrenzen und indem sie sich innerhalb von Beziehungen und Gruppen positionieren. Im Verlauf 
des Sozialisationsprozesses verändert sich die Bedeutung verschiedener Akteure und Kontexte stetig: Während in den ersten Lebensjahren noch die Familie den grössten Einfluss auf die Identitätsentwicklung von Heranwachsenden hat, nimmt dieser Einfluss in der Regel mit zunehmenden Alter ab, während andere Akteure und Institutionen (z. B. Peers, Schule) an Bedeutung gewinnen.

Medien nehmen im Sozialisationsprozess insofern eine besondere Rolle ein, als sie bereits von Beginn an in der Lebenswelt des Kindes präsent sind und es über verschiedene Kontexte und Lebensphasen hinweg begleiten (Bachmair 2007; Tillmann und Hugger 2014; Krotz 2017). Kinder wachsen heutzutage in digitalisierten, multimedialen Umgebungen auf, die zunehmend komplexer, konvergenter und personalisierter werden (Holloway, Green, und Livingstone 2013). Sie nehmen darin schon sehr früh eine aktive Rolle ein, indem sie Medien aus dem existierenden Angebot auswählen sowie über Dauer und Ausgestaltung der Mediennutzung mitentscheiden. Zugleich werden die Zusammensetzung dieses individuellen Medienrepertoires, die Regeln für den Umgang mit Medien und die Erwartungen an die Mediennutzung auch durch andere Akteure - etwa die Eltern - mitbestimmt. Medienerziehung stellt in diesem Zusammenhang ein engeres Konzept als das der Mediensozialisation dar und bezieht sich auf absichtsvolle und meist pädagogisch reflektierte Handlungen mit bestimmten Strategien und Zielen. Der international einflussreichste Ansatz einer Konzeptionalisierung elterlicher Medienerziehung ist die Parental Mediation Theory (Clark 2011), die in den 1990er Jahren von Valkenburg et al. (1999) und Nathanson (1999) massgeblich entwickelt und anhand aktueller Medienentwicklungen in den darauffolgenden Jahren aktualisiert wurde. Während der Erziehungsbegriff konkret das Verhältnis von Erziehenden und zu Erziehenden fokussiert und dabei von einer absichtsgeleiten Einwirkung der älteren auf die jüngere Generation ausgeht, zielt der Begriff der Sozialisation auf die Rolle des Individuums in seiner gesamten materiellen und sozialen Umwelt. Das medienerzieherische Verhalten der Eltern wird aus dieser Perspektive weniger handlungstheoretisch betrachtet, sondern als Teil des familialen Beziehungsgefüges und Interaktionsgeschehens. Welche Rollen Medien im Verlauf des interaktiven Sozialisationsprozesses einnehmen, lässt sich mithilfe zweier Fragen untersuchen: 
Wie lernen Kinder den Umgang mit Medien? Und welche Relevanz haben Medien für den Sozialisationsprozess? (Süss 2004, 65) Da Kinder erstmals in ihrer Familie mit Medien in Kontakt kommen, besitzt diese besonders in den ersten Lebensjahren den stärksten Einfluss sowohl auf die generelle, als auch auf die medienbezogene Sozialisation der Heranwachsenden (Kammerl und Kramer 2016). Die Eltern nehmen dabei eine GatekeeperRolle ein und bestimmen über den Zugang zu und die Nutzung von Medien mit. Zugleich sind sie Vorbilder, Co-Nutzer oder Berater (Spanhel 2006, 113; Jiow et al. 2017; vgl. auch Lampert et al. 2012). So prägt der elterliche Einfluss bereits in der frühen Kindheit spezifische Mediennutzungsmuster, die auch längerfristig stabil bleiben (Vollbrecht 2003, 19) ${ }^{1}$. Medien sind damit Teil alltäglicher familialer Praktiken und der Familienstruktur an sich ("doing family») (Theunert und Lange 2012; Schlör 2016). Zudem ist Mediennutzung ein integraler Bestandteil alltäglicher Kommunikation. Medien vermitteln dabei nicht nur kulturelle Inhalte; Medienrezeption, genauso wie medienbezogene Gefühle und Gedanken sind relevante Konversationsthemen in Familie und Peergroup.

Kommunikation wird in allen Lebensbereichen immer stärker durch zunächst technische und sodann digitale Medien mitbestimmt. Krotz (2007) bezeichnet diesen Metaprozess als Mediatisierung, der in Verbindung mit anderen Transformationsprozessen zu einem umfassenden gesellschaftlichen Wandel führt. Dabei verändern sich nicht nur die Häufigkeit und Dauer der Mediennutzung (quantitativ), sondern auch was und wie mittels welcher Medien kommuniziert wird (qualitativ). Hepp (2018) identifiziert fünf zentrale Trends, die eine tiefgreifende Mediatisierung kennzeichnen: die Ausdifferenzierung der Medientechnologie, deren wachsende Konnektivität, die Omnipräsenz digitaler (Mobil-)Kommunikation, eine beschleunigte Innovationsdichte und die Datafizierung jedweden Medienhandelns. Die Veränderungen auf Ebene der technologischen Entwicklungen und der gesellschaftlichen Gestaltung des Mediensystems

1 Verschiedene Studien weisen zusätzlich auf die Relevanz nicht-medialer Faktoren für die Mediensozialisation hin, etwa des Familienklimas, täglicher Routinen und Anforderungen, individueller Interessen von Kindern und Eltern sowie des mentalen Zustands der Eltern (Wagner, Gebel, und Lampert 2013; Wartberg et al. 2017; Kammerl et. al. 2012; Nathanson 2015; Six, Gimmler, und Vogel 2002). 
(Luhmann 1996) lässt sich als historischer Wandel der Medienumgebung beschreiben. Die tiefgreifende Mediatisierung spiegelt sich auch in den zentralen Bildungs- und Sozialisationsinstanzen wider und wird konkret sichtbar in veränderten Medien und Medienpraktiken in Familien, Schulen und Peergroups. Konkret beobachtbar wird das zunächst durch eine quantitative Bedeutungszunahme der Medien: So wuchs die Zahl der Mediengeräte, die Kindern zur Verfügung stehen, in den letzten Jahren stetig an. Dabei verlagerte sich die Mediennutzung einerseits mehr und mehr in die Kinderzimmer (Süss, Lampert, und Wijnen 2018; Wagner und Lampert 2013). Andererseits wurden digitale Medien zunehmend mobil und damit omnipräsent: In Deutschland besitzen inzwischen 51 Prozent der Kinder zwischen sechs und 13 Jahren und 96 Prozent der Jugendlichen zwischen zwölf bis 19 Jahren ein eigenes Handy oder Smartphone (Feierabend, Rathgeb, und Reutter 2019; Feierabend u. a. 2020). Die starke Verbreitung dieser Geräte hatte auch eine qualitative Bedeutungsverschiebung der Medien zur Folge, was sich in einer zunehmend individualisierten sowie zeitlich und räumlich unabhängigen Nutzung und permanenten Erreichbarkeit und Vernetzung widerspiegelt (Knop et al. 2015; Kühn und Lampert 2015; Subrahmanyam und Greenfield 2008). Aus Sicht der Sozialisationsforschung bleibt die Frage zu klären, wie sich diese Veränderungen von Medienumgebung, -form und -bedeutung auf den Sozialisationsprozess auswirken. Studien zur medienbezogenen Sozialisation nehmen dabei sehr unterschiedliche Aspekte in den Blick. So können auf einer konzeptionellen Ebene folgende vier Untersuchungstypen identifiziert werden:

- Ein Grossteil der Studien fokussiert ein spezifisches Medium oder einen expliziten Medieninhalt und dessen Relevanz für kindliche Sozialisation. Oft stehen dabei das Fernsehen (z. B. Theunert und Gebel 2000; Wegener 2008), das Lesen (eine Übersicht hierzu bietet Wieler 2010) oder das Radiohören (beispielsweise Hoffmann 2002; Paus-Haase, Aufenanger und Mattusch 2000) im Mittelpunkt. In den vergangenen Jahren wurde ausserdem die Bedeutung einzelner digitaler Medien, beispielsweise die von Videospielen (Fromme, Meder und Vollmer 2000; Lampert et al. 2012) oder des Internets (Livingstone und Haddon 2009; Mascheroni und Cuman 2014), untersucht. Nur wenige Studien nehmen dagegen eine multimediale Perspektive ein und beziehen 
das Phänomen der Medienkonvergenz mit ein (Paus-Hasebrink und Kulterer 2014; Paus-Hasebrink 2017; Schuegraf 2008, 2010; Wagner und Theunert 2006).

- Daneben beschäftigt sich ein Teil der Forschung mit spezifischen Sozialisationsthemen, wie etwa der Entwicklung einer medialen Identität (z. B. Hugger 2010; Lampert et al. 2012; Mikos, Hoffmann und Winter 2007; Tillmann 2008). Diese Untersuchungen fokussieren sich auf Teilbereiche der Sozialisation und diskutieren die Ergebnisse nicht im Hinblick auf die Konsequenzen für den Sozialisationsprozess an sich.

- Eine dritte Art von Studien betrachtet Medien in spezifischen Kontexten oder Konstellationen, beispielsweise in der Familie oder der Peergroup. Während viele dieser Untersuchungen die elterliche Medienerziehung bzw. parental mediation in den Blick nehmen (z. B. Connell, Lauricella, und Wartella 2015; Schaan und Melzer 2015; Vaala und Bleakley 2015; Valkenburg et al. 2013; Wagner, Gebel, und Lampert 2013; Pfetsch 2018), gibt es nur wenige, die den Einfluss der Medienentwicklung auf die Familienstruktur, die Kommunikation innerhalb der Familien oder die Familienbeziehungen und die damit verbundenen Konsequenzen für Sozialisation und kindliche Entwicklung untersuchen (Mesch 2006; Wagner, Gebel, und Lampert 2013; Schlör 2016).

- Schliesslich existieren im Bereich der Mediensozialisationsforschung vereinzelt Studien, die den Fokus auf die Bedeutung medialer Praktiken innerhalb eines spezifischen sozialen Milieus legen (z. B. PausHasebrink, Kulterer, und Sinner 2019; Paus-Hasebrink 2017; PausHasebrink und Kulterer 2014).

Wie dieser konzeptionelle Überblick zeigt, herrscht vor allem ein Mangel an verstehenden Perspektiven auf die Rolle der sich ändernden Medienumgebung für die Sozialisation. Mit Ausnahme der von Ingrid Paus-Hasebrink koordinierten Studie zu sozial benachteiligten Familien (in Österreich) (ebd.) gibt es kaum Projekte, die das Zusammenspiel verschiedener Medientypen, unterschiedlicher Medienthemen sowie der Sozialisation in den Blick nehmen. Darüber hinaus fehlt der Vergleich unterschiedlicher sozialer Domänen und Milieus. Ein derart breiter Fokus wird allerdings benötigt, um die Konsequenzen komplexer gesellschaftlicher Transformationen für die Heranwachsenden zu verstehen. 


\section{Methodologische Zugänge der Sozialisationsforschung}

Neben inhaltlichen Fragen sind im Rahmen der Sozialisationsforschung auch methodologische Entscheidungen zu fällen. Um das Wechselspiel zwischen individuellen und sozialen Bedingungen für die Mediennutzung als Ausgangspunkt der Untersuchung von Sozialisationsprozessen zu bestimmen, knüpfte die Mediensozialisationsforschung lange Zeit an zwei Traditionen an, die auf den Annahmen des Pragmatismus und des symbolischen Interaktionismus basieren (Charlton und Bachmair 1990): Die eine Tradition nimmt die phänomenologische Konzeption der sozialen Welt (Schütz 1974) als Ausgangspunkt, die andere nimmt primär Bezug auf Georg Herbert Meads Theorie von Subjekt und Gesellschaft (Mead 1968) sowie deren Weiterentwicklung im Kontext des Symbolischen Interaktionismus. Ziel beider Theorielinien ist es, die Bedeutung und die Struktur der sozialen Welt $\mathrm{zu}$ verstehen und die Bedeutung von Kommunikationsprozessen zu rekonstruieren. Entsprechend der Zuordnung zu einer der beiden Traditionslinien wurden in der (Medien-)Sozialisationsforschung unterschiedliche methodologische Herangehensweisen genutzt: Um die Entstehung sozialer Ordnung im Alltag zu verstehen, werden das Alltagslebens als Herstellungsleistung, also «doing everyday life» im Hier und Jetzt - ebenso, wie 《doing family» (Jurczyk, Lange, und Thiessen 2014) oder «doing gender» (West und Zimmerman 1987) - mit Methoden der Cultural Studies und der Ethnografie untersucht. Im Sinne einer rekonstruktiv verfahrenden Sozialforschung wurden aber auch Ansätze entwickelt, die sich eher an der Rekonstruktionslogik der Objektiven Hermeneutik nach Oevermann orientieren und versuchen, die sozial geteilte Bedeutung von Handlungen zu analysieren. So orientierte sich die strukturanalytische Rezeptionsforschung von Charlton und Neumann-Braun (1986) methodologisch an hermeneutisch-rekonstruktiven Verfahren. Sie untersucht die Bedeutung der Mediennutzung als soziale Handlungssituation und rückt ihre Rolle und Funktion für das soziale Bezugssystem in den Mittelpunkt. In der Theorie der Medienaneignung (Schorb und Theunert 2000) steht deren Bedeutung für die individuellen Biografien im Mittelpunkt. Hier wird die aktive Auseinandersetzung mit dem Medienangebot im lebensweltlichen Kontext beleuchtet und die Integration der Medien in die eigene Lebensbiografie 
erklärt. Eine ähnliche Ausrichtung hat auch das Konzept der «handlungsleitenden Themen» in der Medienforschung (Bachmair 1994).

In diesen Ansätzen wurde dabei bereits versucht, die verschiedenen Ebenen der gesellschaftlichen Struktur der Medienkommunikation zu berücksichtigen. Für das Verständnis der Medienpraktiken ist eine Verknüpfung der Mediennutzung auf der Mikroebene mit den Funktionen des Mediensystems auf der Makroebene erforderlich. Im Anschluss an die Beiträge von Habermas und Luhmann wurden Massenmedien in erster Linie in ihrer Funktion für Systeme wie Politik und Ökonomie betrachtet. Die Systeme scheinen zunehmend die Lebenswelten zu kolonialisieren. Sozialisationsforschung muss folglich das Zusammenspiel von Lebenswelt und Systemen betrachten. Habermas ging davon aus, dass neben den symbolisch generalisierten Kommunikationsmedien Macht und Geld, auch Massenmedien beitragen, die Handlungsdispositive der Individuen auszurichten.

«Es scheint so, als erhielten die Systemimperative nun über die mediatisierte Familie hinweg die Chance eines unmittelbaren, durch das weiche Medium der Massenkultur allenfalls gebremsten Zugriffs auf das intrapsychische Geschehen.» (Habermas 1995, 568).

Dabei blieben aber die Möglichkeiten der Datenökonomie im digitalen Zeitalter freilich noch unberücksichtigt.

Ausgehend vom französischen Strukturalismus sind auch poststrukturalistische Ansätze hinzugetreten, die in jüngerer Zeit hinsichtlich ihrer Potentiale für eine medienpädagogische (Sozialisations-)Forschung diskutiert werden (Lüders 2007; Münte-Goussar 2016; Bettinger 2016; Dander 2017). Analog zur Rezeption in der erziehungswissenschaftlichen Diskussion wird die Adaption von Poststrukturalismus und Diskursanalyse verwendet, um subjekttheoretische Bestimmungen von Autonomie und Handlungskompetenz zu hinterfragen. Im Anschluss an Wrana et al. (2014) können die hierzu vorliegenden Arbeiten unterschieden werden in Analysen klassischer spezialdiskursiver Formationen, gouvernementalitätstheoretische Studien, Subjektivierungsregime und Subjektivationen, sowie disziplinäre Selbstreflexivierungen. Deren Nutzbarmachung für die Forschung steht jedoch weitgehend noch aus. So sind 
«medienpädagogische [...] Arbeiten, die sich auf diskurstheoretische Annahmen stützen oder mit unterschiedlichen diskursanalytischen Instrumentarien arbeiten zum einen spärlich gesät und zum anderen eher an den Rändern der erziehungswissenschaftlichen Teildisziplin angesiedelt» (Dander 2017, 51).

Mit der Situationsanalyse (vgl. Clarke 2012) liegt ein methodologischer Vorschlag vor, der zwischen (Neo-)Pragmatismus und (Post-)Strukturalismus vermittelt.

Daneben existieren gerade für die Beschreibung dynamischer Entwicklungen der Strukturen des menschlichen Zusammenlebens jenseits der soziologischen Megaparadigmen (Diaz-Bone 2012) weitere Ansätze, die für Forschung zur Sozialisation in zunehmend mediatisierten Gesellschaften fruchtbar gemacht werden können. Die von Norbert Elias geprägte Figurations- und Prozesstheorie (1971) wird in den letzten Jahren wieder verstärkt rezipiert (Treibel 2009; Hepp und Hasebrink 2014). Dies machte etwa der Call for Papers zur Herbsttagung der Sektion Medienpädagogik der DGfE im Jahr 2018 deutlich, indem er explizit die Thesen zur «tiefgreifende Mediatisierung» des 〈Communicative Figurations〉 Research Network der Universitäten Bremen und Hamburg aufgriff (Hepp und 〈Communicative Figurations〉 research network 2017). Der Ansatz der kommunikativen Figurationen (Hepp und Hasebrink 2014; Hepp, Breiter, und Hasebrink 2018) knüpft an den Figurationsbegriff an und entwickelt ihn für die medien- und kommunikationswissenschaftliche Forschung weiter.

\section{Das Konzept der kommunikativen Figurationen als theoretische Rahmung von Sozialisationsprozessen}

Grundlage des Ansatzes der kommunikativen Figurationen ist der von Norbert Elias im Rahmen seiner Prozesssoziologie geprägte Begriff der Figuration (Elias 1971). Darunter ist ein Interdependenzgeflecht zu verstehen, innerhalb dessen Akteure (Individuen, Gruppen usw.) miteinander interagieren und gemeinsam ihre soziale Umwelt gestalten. In Grösse und Form können diese Netzwerke dabei beliebig variieren, also sowohl aus lediglich zwei Akteuren (z. B. in Paarbeziehungen), aus vergleichsweise 
überschaubaren Gruppen (wie Familien) oder aus komplexen Gesellschaften bestehen. Bezogen auf erziehungswissenschaftliche Fragestellungen wären etwa ganze Schulsysteme, Schulen, Klassenverbünde, Peer-Gemeinschaften oder Mediatisierungsgemeinschaften informellen Lernens figurationstheoretisch $\mathrm{zu}$ fassen (Wolf und Wegmann 2020). Indem das Konzept der Figurationen vor allem die Beziehungen zwischen den Akteuren fokussiert und damit sowohl die Mikro- als auch die Makroebene einbezieht, hebt es die traditionelle Unterscheidung von Individuum und Gesellschaft auf und erkennt an, dass beide nur zusammen gedacht und beschrieben werden können. Damit hat Elias ein «einfaches aber effizientes Werkzeug» (Elias 1971, 141) für die Untersuchung sozialer Phänomene geschaffen (Kammerl et al. 2020, 381f.).

Ausgangspunkt für die Weiterentwicklung des Figurationsansatzes ist die Annahme einer tiefgreifenden Mediatisierung, in deren Zuge (digitale) Medien vermehrt soziale Zusammenhänge - und damit auch kindliche Lebenswelten - prägen (Hepp und 〈Communicative Figurations〉 research network 2017). Verbunden mit der Prämisse, dass bei der gemeinschaftlichen Konstruktion sozialer Zusammenhänge (Figurationen) den dabei eingesetzten kommunikativen Praktiken eine zentrale Rolle zukommt, erscheint eine Auseinandersetzung mit digitalen Medien im Rahmen der Sozialisationsforschung wichtig. Als zentrale Elemente der somit kommunikativ hergestellten (also: kommunikativen) Figurationen machen Hepp und Hasebrink (2014) neben der Akteurskonstellation und einem spezifischen Relevanzrahmen auch die Medienensembles innerhalb der Figurationen und die mit diesen verbundenen kommunikativen Praktiken aus. Damit erlaubt das Konzept, sowohl das individuelle Medienrepertoire der beteiligten Akteure (also der einzelnen Familienmitglieder) als auch das personenübergreifende Medienensemble einer Figuration (in diesem Fall: der Familie) in die Analyse einzubeziehen und beide zueinander in Beziehung zu setzen (Kammerl et al. 2020, 383). Vor dem Hintergrund sozialer und technologischer Entwicklungen sowie aufgrund «fluktuierender Machtbalancen» (Elias 1971, 143) zwischen den beteiligten Akteuren sind die Beziehungsnetzwerke kontinuierlichen Veränderungsprozessen unterworfen. Darüber hinaus prägen auch affektive Bindungen (Valenzen) die Beziehungen zwischen den Akteuren. Insofern können kommunikative 
Figurationen nicht als starre Gebilde gesehen, sondern müssen stets sowohl in ihrer Prozesshaftigkeit als auch ebenenübergreifend, also unter Einbeziehung individueller und geteilter Aspekte, betrachtet werden.

Durch seine spezifische Perspektive, die Einbeziehung unterschiedlicher Aspekte sozialer Phänomene und seine theoretische und methodologische Anschlussfähigkeit ist der Ansatz geeignet, die Sozialisationsforschung zu bereichern.

\section{Sozialisationsprozesse innerhalb kommunikativer Figurationen}

Die Erforschung mediatisierter Sozialisationsprozesse ist an eine Reihe methodologischer Voraussetzungen geknüpft. Stehen dabei kommunikative Figurationen im Zentrum der Analyse, ergeben sich hieraus weitere Bedingungen für das empirische Vorgehen, die im Folgenden anhand der konstituierenden Merkmale aufgezeigt werden: Erstes kennzeichnendes Merkmal der Sozialisation ist ihre lebenslange Dauer. Da das Konzept die Entwicklung von Menschen als Wachsen innerhalb einer Gesellschaft umfasst, ist sein Prozesscharakter zentral und sollte die empirische Forschung leiten. Die Prozesshaftigkeit findet sich analog in der Konzeption (kommunikativer) Figurationen wieder. Sich ständig wandelnde Valenzen und fluktuierende Machtbalancen kennzeichnen dabei den Figurationsstrom. Sie stellen für die Analyse von Sozialisationsprozessen in unterschiedlichen sozialen Domänen wertvolle Analyseeinheiten dar. So verspricht eine detaillierte Untersuchung von Veränderungen der Beziehungen innerhalb einer Figuration (hier der Familie) Einblicke auch in kindliche Entwicklungsprozesse. Damit ermöglicht der Zugang etwa Beobachtungen im Hinblick auf die oben bereits als bedeutsam charakterisierte Dimension von Zugehörigkeit und Abgrenzung. Richtet sich das Augenmerk hingegen auf fluktuierende Machtbalancen, stehen Kontrolle und Autonomie stärker im Mittelpunkt der Analyse. Auch in dem von Elias eingesetzten Begriff der Machtbalance spiegelt sich die Prozessperspektive wider: Elias versteht Macht nicht als eine den einzelnen Akteuren zuzuschreibende Ressource, sondern als ein Gleichgewicht, in dem jeder Handlungsmacht eine entgegengesetzte Macht anderer Akteure zukommt Unabhängig davon, 
welche Analysedimension die Auseinandersetzung mit Sozialisation leitet, scheint es nicht zuletzt aufgrund der Konzeption des Aufwachsens zentral, längsschnittlich konzipierte Analysen solchen vorzuziehen, die nur einen Zustand in den Blick nehmen. Diese tragen dann auch den Veränderungen von Beziehungen und Machtbalancen innerhalb der Figurationen Rechnung. Sozialisation ist darüber hinaus durch Interaktion gekennzeichnet. Entsprechend sollte ihre empirische Erforschung auch die Sichtweisen aller beteiligten Akteure einbeziehen. Der Ansatz der kommunikativen Figurationen berücksichtigt über die Akteurskonstellation die Gesamtheit der Akteure innerhalb einer Figuration (z. B. der Figuration Familie). Neben Familienmitgliedern im engeren Sinne kann diese auch weitere Personen umfassen, die am Familienalltag beteiligt sind. Darüber hinaus bezieht die Akteurskonstellation die Beziehungen zwischen den Familienmitgliedern mit ein. Da Veränderungen in den Rollen einzelner Personen einer Figuration und der Wandel der Beziehungen zwischen den einzelnen Akteuren von diesen oftmals nicht bewusst wahrgenommen werden, gilt es Forschungsprozesse so zu gestalten, dass der Zugang zu entsprechenden Entwicklungen möglichst breit angelegt ist und Reflexionsanlässe bietet. Die Kombination mehrerer Strategien scheint hierfür besonders geeignet: Zum einen erlaubt die Einbeziehung verschiedener Perspektiven unterschiedliche, sich stützende oder ergänzende (aber auch einander widersprechende) Sichtweisen auf ein Phänomen. Zum anderen bieten sich vor allem Verfahren qualitativer Sozialforschung an, um den untersuchten Personen den Raum und die Möglichkeiten zu geben, Veränderungen zu reflektieren und zu rekonstruieren. Der Mediatisierung des Familienalltags und der Sozialisation sowie der damit einhergehenden zentralen Bedeutung von Medien und Medienpraktiken für die kindliche Lebenswelt trägt der Ansatz kommunikativer Figurationen Rechnung: Zum einen bezieht er das Medienensemble der Familie (also die Gesamtheit aller den Familienmitgliedern gemeinsam zur Verfügung stehender Medienangebote) mit ein und berücksichtigt auch dessen Wandel. Zum anderen nimmt der Ansatz auf das individuelle Medienrepertoire (hier: des Kindes) Bezug, über das sich erfassen lässt, welche Rolle die Medien im Alltag des Kindes und im Sozialisationsprozess tatsächlich spielen. Dabei umfasst das Medienrepertoire alle Medienangebote, die das Kind normalerweise in seine Medienpraktiken einbezieht und 
die Art und Weise, wie unterschiedliche Medien kombiniert werden. Das Medienrepertoire bezeichnet also ein relativ stabiles, individuelles und medienübergreifendes Muster der Medienpraktiken, das die Individuen orientiert an übergreifenden Prinzipien (z. B. Nützlichkeit, Involvement, Effektivität) entwickeln (Hasebrink und Hölig 2017)². Beide Perspektiven sollten im Rahmen empirischer Analysen zu mediatisierten Sozialisationsprozessen berücksichtigt werden. Der Medienrepertoireansatz ermöglicht im Wechselspiel von Vergesellschaftung und Entwicklung der Handlungsfähigkeit von Kindern und Jugendlichen ein relationales Verständnis, indem es die Verschränkung des Individuums mit dem zur Verfügung stehenden Medienensemble, der jeweiligen kommunikativen Figuration und der sich wandelnden Medienumgebung betrachtet. Dass Individuen vielfältig keine Hoheit über ihre Daten haben und die Ziele und Funktionsweisen der Algorithmen, welche die Interaktion der benutzten informatischen Systeme steuern, verbindet sich in der Analyse mit den Akteurskonstellationen und deren Machtbalancen und Valenzen. Auf der Makroebene ist hierbei die Handlungsfähigkeit von Staaten bei der Durchsetzung von rechtlichen und politischen Regeln z. B. bei Datenschutz und Jugendschutz im Kontext globaler Entwicklungen einbezogen. Auf Mikro- und Mesoebene umfasst es die Fähigkeit des Einzelnen, seine Medienpraktiken im Kontext von sozialen und technischen Zwängen selbst zu bestimmen.

Damit Ensemble und Repertoire tatsächlich angemessen erfasst werden, bietet es sich an, nicht nur auf Interviewdaten zuzugreifen, sondern die genutzten Medien beispielsweise vor Ort in Augenschein zu nehmen oder fotografisch zu dokumentieren bzw. durch die Untersuchten dokumentieren zu lassen. Indem diese so über Auswahl und Gewichtung der Interviewthemen mitbestimmen, wird ihre Rolle als gleichberechtigte Interaktionspartner im Forschungsprozess unterstrichen. Vielversprechend - wenngleich mit Blick auf das Thema Datenschutz voraussetzungsvoll - erscheint auch der Zugang über die tatsächlichen Medienpraktiken der Akteure, indem diese beispielsweise direkt beobachtet oder ebenfalls fotooder video-dokumentiert werden. Dabei können aus den Beobachtungen

2 Paus-Hasebrink und Hasebrink (2014) schlagen vor, dabei die Gesamtheit aller medialen und nicht-medialen kommunikativen Handlungen eines Individuums in den Blick zu nehmen und den Ansatz des Medienrepertoires auf ein Kommunikationsrepertoire zu erweitern. 
bzw. Fotos Anlässe zur Reflexion und zu vertiefenden Gesprächen über die Bedeutung von Medien und Medienhandeln entstehen. Eine entsprechende Erhebung unterschiedlicher Datentypen hat sich insbesondere beim Blick auf familiale Sozialisation und im Umgang mit Kindern bereits in früheren Forschungsprojekten als sehr bereichernd erwiesen (Paus-Hasebrink et al. 2017; Paus-Haase und Schorb 2000). Die Notwendigkeit, auch Veränderungen der Medienlandschaft zu erfassen und zu berücksichtigen, liefert darüber hinaus ein weiteres Argument für eine längsschnittliche Konzeption der Analyse.

Im medienpädagogischen Theoriediskurs wird zudem die dichotome Trennung im Mensch-Technik-Verhältnis kritisch reflektiert. Dass Individuen keine Hoheit über ihre Daten haben und die Ziele und Funktionsweisen der Algorithmen, welche die Interaktion der benutzten informatischen Systeme steuern, immer weniger durchschauen können, findet in der einseitig auf Kompetenzen orientierten Bildungsforschung und einer Sozialisationsforschung, die ebenso einseitig das produktiv realitätsverarbeitende Subjekt betont, zu wenig Beachtung. Neue, sich im Mediatisierungsprozess entwickelnde Machtgefälle bzw. die sich damit verändernden Machtdispositive im Medienzusammenhang müssen aber in der aktuellen Forschung stärker aufgegriffen werden. Der Medienrepertoireansatz legt ein relationales Verständnis von Medienkompetenz und Medienbildung nahe, indem er die Verschränkung des Individuums mit dem zur Verfügung stehenden Medienensemble der jeweiligen kommunikativen Figuration und der sich wandelnden Medienumgebung zu betrachten erlaubt. Den Ausgangspunkt der Analyse stellen die Medienpraktiken dar, die jedoch durch die Berücksichtigung ihrer Eingebundenheit in Akteurskonstellationen damit verbundene Machtbalancen und Valenzen erweitert wird.

Schliesslich gilt es zu berücksichtigen, dass Heranwachsende im Laufe ihrer Sozialisation verschiedene handlungsleitende Themen berarbeiten und dabei auch auf symbolisches Material zurückgreifen, das ihnen $\mathrm{Me}$ dien bieten (Bachmair 1994; Paus-Haase et al. 2004; Wegener 2016). Hierbei kann es sich um klassische Entwicklungsthemen bzw. -aufgaben handeln (z. B. Havighurst 1972), denen sich gemeinhin alle Heranwachsenden gegenübersehen, aber auch situationsbezogene Themen, mit denen sich Kinder in ihrem jeweiligen Lebensalltag (ausgelöst etwa durch kritische 
Lebensereignisse) befassen. Die Annahme, dass handlungsleitende Themen nicht nur die Kinder, sondern die gesamte Familie (als kommunikative Figuration) beschäftigen, greift der Ansatz kommunikativer Figurationen mit dem Relevanzrahmen ${ }^{3}$ auf, der jede Figuration prägt. In der empirischen Sozialisationsforschung kann dem Relevanzrahmen nur mit einem offenen, rekonstruktiven Zugang, also mit qualitativen Verfahren, angemessen Rechnung getragen werden.

\section{Fazit}

Der Ansatz der kommunikativen Figurationen erscheint vor dem Hintergrund einer tiefgreifenden Mediatisierung als ein geeigneter theoretischer Rahmen für die Untersuchung von Sozialisationsprozessen. Er berücksichtigt zentrale sozialisationstheoretische Elemente sowie in besonderer Weise die Bedeutung medienvermittelter Kommunikation für die familiale Beziehungsgestaltung.

So wird etwa die in vielen Studien vorgenommene Beschränkung auf einzelne Medienangebote überwunden, indem sowohl das gesamte Medienensemble innerhalb einer Figuration als auch die individuellen Medienrepertoires der einzelnen Figurationsmitglieder einbezogen und zueinander in Beziehung gesetzt werden. Zugleich weist der Ansatz eine rein technikzentrierte Perspektive zurück und fokussiert stattdessen auf die Kommunikation und insbesondere auf die sozialen Aushandlungsprozesse zwischen den Familienmitgliedern unter den Bedingungen des familialen Medienensembles sowie des individuellen Medienrepertoires. Zwar beinhalten digitale Medien Merkmale, mit denen sie mögliche Kommunikation vorstrukturieren. Die Gestaltung dieser Möglichkeiten in den verschiedenen sozialen Domänen (z. B. Familien, Schulen, Peer Group) erklärt sich aber nicht aus einer eigenen Medienlogik allein, sondern muss vielmehr

3 In der Konzeption der Sozialisation als produktive Auseinandersetzung mit eigenen Anlagen und der umgebenden Umwelt kommt den Aufwachsenden eine aktive Rolle zu. Entsprechend sollten u. E. ihre Perspektive auf den Relevanzrahmen im Vordergrund stehen. Gleichwohl sind aber auch Themen denkbar, die die Familie als Figuration beschäftigen (z. B. die Arbeitslosigkeit eines Elternteils) oder für einzelne andere Familienmitglieder handlungsleitend und für die Kinder relevant sind. Auch diese sollten in der Untersuchung von Sozialisationsprozessen Berücksichtigung finden. 
aus der Verfasstheit der jeweiligen Akteurskonstellation und der Einbettung der Medien in die Gesamtheit des Medienensembles dieser Domänen erfolgen. Es ist daher richtig zu betonen, dass z. B. Smartphones dezentrierte Interaktionsordnungen mit raumexternen Kommunikationspartnern ermöglichen und neue Formen synchroner und asynchroner Kommunikation mit sich bringen (z. B. Wiesemann und Fürtig 2018, 208). Welche Möglichkeiten aber mit welchen Kommunikationspartnern von Kindern tatsächlich in Familien, Schulen oder beim Austausch mit Peers genutzt werden, ist ohne Kenntnis der entsprechenden Beziehungsgeflechte jedoch kaum verstehbar. Ebenso lässt sich Attraktivität eines Medienangebots im Medienensemble nicht ohne die medialen und non-medialen Alternativen bewerten. Auch wenn im Rahmen des «Neuen Materialismus in der Kindheitsforschung» (Balzer und Huf 2019) die «Pädagogik der Dinge» (Nohl und Wulf 2013) wieder stärker in den Vordergrund gerückt werden, wird damit kein Rückfall in technikdeterministische Sichtweisen gestützt, sondern vielmehr versucht, das sozialkonstruktivistische Verständnis von Kindheit um die Berücksichtigung der (trans)formativen Kraft der materiellen Welt zu erweitern. Medien werden deshalb auch in dem vorliegenden Ansatz weder als eine unabhängige Sozialisationsinstanz noch als ein zentrales, eigenständiges Entwicklungsfeld betrachtet, sondern als ein Mittel der Kommunikation. Die zunehmende Verbreitung und Ausdifferenzierung digitaler Medien sind Merkmale tiefgreifender Mediatisierung und verändern den gesamten Prozess des Aufwachsens, inklusive des Handelns jedes einzelnen Familienmitgliedes. Die Medienpraktiken als soziales Handeln der einzelnen Individuen stellen den Ausgangs- und Mittelpunkt der Analyse dar. Zugleich wird jedoch auch die Familie als Ganzes mithilfe der Akteurskonstellation sowie der damit verbundenen Machtbalancen und Valenzen in die Betrachtung einbezogen.

Über individuelle und gemeinsame Medienpraktiken schaffen und teilen die Familienmitglieder Bedeutungen. Auf diese Weise stellen sie ihren Alltag, die Familie an sich und das Aufwachsen innerhalb dieser permanent her und eignen sich Medien als Techniken und Handlungspraktiken, aber auch als Inhalte an. Über die Feststellung dieser kommunikativen Praktiken hinaus ermöglicht der Ansatz der kommunikativen Figurationen jedoch tiefergehende Betrachtungen medienbezogener Sozialisation. 
So prägen die handlungsleitenden Themen der einzelnen Individuen das soziale Miteinander ebenso, wie der Relevanzrahmen der gesamten Figuration. Gleichzeitig beschränkt sich der Ansatz nicht auf wenige Elemente der Sozialisation. Vielmehr nimmt er, vermittelt über fluktuierende Machtbalancen und sich wandelnde Beziehungen innerhalb der Figuration, Sozialisation als dynamischen Prozess in den Blick. Die in diesem Beitrag aufgezeigten methodologischen Implikationen verweisen darauf, dass Forschung im Kontext mediatisierter Sozialisation idealerweise als eine (qualitative) Längsschnittanalyse angelegt sein sollte, in die die Perspektive mehrerer (bestenfalls aller) Figurationsmitglieder einfliesst und die über die Einbeziehung verschiedener Datentypen einen breiten Zugang zum Feld sucht. Je nach Auswertungsmethode (z. B. qualitative Inhaltanalyse, Dokumentarische Methode, Grounded Theory etc.) lassen sich fallspezifische als auch fall-übergreifende Aussagen im Hinblick auf den Sozialisationsprozess formulieren. Je länger dieser begleitet werden kann, desto valider die Daten und grösser ihr Erklärungspotenzial.

Eine Analyse mediatisierter Sozialisation, die Sozialisationskontexte als kommunikative Figurationen konzipiert und diese zum Ausgangspunkt ihrer Forschung macht, scheint daher geeignet, die bisher dominierenden und scheinbar weit voneinander entfernt agierenden Theoriestränge teilweise zusammenzubinden und für die medienpädagogische Forschung fruchtbar zu machen. 


\section{Literatur}

Bachmair, Ben. 2007. «Mediensozialisation. Entwicklung von Subjektivität in medialen und kulturellen Figurationen». In Mediensozialisationstheorien. Neue Modelle und Ansätze in der Diskussion, herausgegeben von Dagmar Hoffmann und Lothar Mikos, 67-91. Wiesbaden: VS, Verlag für Sozialwissenschaften. http://www.urn.fi/urn:nbn:de:hebis:34-2009081729502.

Bachmair, Ben. 1994. «Handlungsleitende Themen: Schlüssel zur Bedeutung der bewegten Bilder für Kinder». In Handbuch Medienerziehung im Kindergarten, herausgegeben von Christine Feil, Ulf Lehnig, H. G. Beisenherz, und Maria Furtner-Kallmünzer, 1, Pädagogische Grundlagen:171-84. Opladen: Leske + Budrich Verlag. http://www.urn.fi/urn:nbn:de:hebis:34-2009040826862.

Balzer, Nicole, und Christina Huf. 2019. «Kindheitsforschung und Neuer Materialismus». In Handbuch Philosophie der Kindheit, herausgegeben von Johannes Drerup und Gottfried Schweiger. 50-58. J. B. Metzler, Stuttgart. https://doi. org/10.1007/978-3-476-04745-8_7.

Bettinger, Patrick. 2016. Mediale Diskurse und biographische Transformationen. In Mediale Diskurse, Kampagnen, Öffentlichkeiten, herausgegeben von Johannes Fromme, Florian Kiefer und Jens Holze, 9-33. Wiesbaden: Springer Fachmedien Wiesbaden. https://doi.org/10.1007/978-3-658-10526-6_2.

Charlton, Michael, und Ben Bachmair. 1990. Medienkommunikation im Alltag: interpretative Studien zum Medienhandeln von Kindern und Jugendlichen. Schriftenreihe / Internationales Zentralinstitut für das Jugend- und Bildungsfernsehen 24. München: Saur. http://www.urn.fi/urn:nbn:de:hebis:34-2007100819326.

Charlton, Michael, und Klaus Neumann-Braun. 1986. Medienkonsum und Lebensbewältigung in der Familie: Methode und Ergebnisse der strukturanalytischen Rezeptionsforschung, mit fünf Falldarstellungen. München: Psychologie Verlags Union.

Clark, Lynn S. 2011. «Parental Mediation Theory for the Digital Age». Communication Theory. Vol. 21 (4): 323-343.

Clarke, Adele E. 2012. Situationsanalyse: Grounded Theory nach dem Postmodern Turn. Interdisziplinäre Diskursforschung. Wiesbaden: Springer VS.

Connell, Sabrina L., Alexis R. Lauricella, und Ellen Wartella. 2015. Parental CoUse of Media Technology with Their Young Children in the USA. Journal of Children and Media 9 (1): 5-21. https://doi.org/10.1080/17482798.2015.997440.

Dander, Valentin. 2017. «Medien - Diskurs - Kritik. Potenziale der Diskursforschung für die Medienpädagogik». MedienPädagogik: Zeitschrift für Theorie Und Praxis Der Medienbildung 27 (Spannungsfelder \& blinde Flecken):50-64. https://doi.org/10.21240/mpaed/27/2017.01.15.X.

Diaz-Bone, Rainer. 2012. «Review Essay: Situationsanalyse - Strauss meets Foucault?». Forum Qualitative Sozialforschung / Forum: Qualitative Social Research Vol 14 (November): No 1 (2013). https://doi.org/10.17169/fqs-14.1.1928.

Elias, Norbert. 1971. Was ist Soziologie? 1. Aufl. Grundfragen der Soziologie. Weinheim: Juventa-Verl. 
Feierabend, Sabine, Thomas Rathgeb, Hediye Kheredmand, und Stephan Glöckler. 2020. «JIM-Studie 2020. Jugend, Information, Medien. Basisuntersuchung zum Medienumgang 12- bis 19-Jähriger». Herausgegeben von Medienpädagogischer Forschungsverbund Südwest (mpfs). Medienpädagogischer Forschungsverbund Südwest (LFK, LMK). https://www.mpfs.de/fileadmin/files/ Studien/JIM/2020/JIM-Studie-202O_Web_final.pdf.

Feierabend, Sabine, Thomas Rathgeb, und Theresa Reutter. 2019. «KIM-Studie 2018. Kindheit, Internet, Medien. Basisstudie zum Medienumgang 6- bis 13-Jähriger in Deutschland». Herausgegeben von Medienpädagogischer Forschungsverbund Südwest (mpfs). Medienpädagogischer Forschungsverbund Südwest (LFK, LMK). https://www.mpfs.de/fileadmin/files/Studien/KIM/2018/ KIM-Studie_2018_web.pdf.

Fromme, Johannes, Norbert Meder, und Nikolaus Vollmer. 2000. Computerspiele in der Kinderkultur. Virtuelle Welten 1. Opladen: Leske + Budrich.

Habermas, Jürgen 1995. Theorie kommunikativen Handelns. Band 2: Zur Kritik der funktionalistischen Vernunft. (Erstveröff. 1981). Frankfurt a. M. Suhrkamp.

Havighurst, Robert J. 1972. Developmental Tasks and Education,. 3. Aufl. New York: Addison-Wesley Longman Ltd.

Hasebrink, Uwe, und Sascha Hölig. 2017. «Deconstructing Audiences in Converging Media Environments». In Media Convergence and Deconvergence, herausgegeben von S. Sparviero, C. Peil, und G. Balbi. 113-133. Cham: Springer International Publishing.

Hepp, Andreas. 2016. «Kommunikations- und Medienwissenschaft in datengetriebenen Zeiten». Publizistik 61 (3): 225-46. https://doi.org/10.1007/s11616-0160263-y.

Hepp, Andreas, Andreas Breiter, und Uwe Hasebrink. 2018. Communicative Figurations. Cham: Springer International Publishing. http://link.springer. com/10.1007/978-3-319-65584-0.

Hepp, Andreas, und "Communicative Figurations" research network. 2017. «Transforming Communications. Media-related Changes in Times of Deep Mediatization». Working Paper 2017. 〈Communicative Figurations〉 research network, ZeMKI, Centre for Media, Communication and Information Research. https://doi.org/10.1007/978-3-319-65584-0_2.

Hepp, Andreas, und Uwe Hasebrink. 2014. «Kommunikative Figurationen - ein Ansatz zur Analyse der Transformation mediatisierter Gesellschaften und Kulturen». In Von der Gutenberg-Galaxis zur Google-Galaxis: alte und neue Grenzvermessungen nach 50 Jahren DGPUK, herausgegeben von Birgit Stark, Oliver Quiring, Nikolaus Jackob und Deutsche Gesellschaft für Publizistikund Kommunikationswissenschaft, 343-60. Schriftenreihe der Deutschen Gesellschaft für Publizistik- und Kommunikationswissenschaft, Band 41. Konstanz München: UVK Verlagsgesellschaft. 
Hoffmann, Dagmar. 2002. «Radionutzung von Jugendlichen. Individualisiertes Alltagshandeln oder Selbstsozialisation». In Wozu Jugendliche Musik und Medien gebrauchen: jugendliche Identität und musikalische und mediale Geschmacksbildung, herausgegeben von Renate Müller, 84-97. Jugendforschung. Weinheim: Juventa.

Holloway, Donell, Lelia Green, und Sonia Livingstone. 2013. Zero to Eight: young children and their internet use. LSE London, EU Kids Online. http://eprints.lse. ac.uk/id/eprint/52630.

Hugger, Kai-Uwe. 2010. Digitale Jugendkulturen. 1. Aufl. Wiesbaden: VS Verlag für Sozialwissenschaften. https://doi.org/10.1007/978-3-531-91908-9.

Hurrelmann, Klaus, und Ulrich Bauer. 2015. «Das Modell der produktiven Realitätsverarbeitung in der aktuellen Diskussion». ZSE Zeitschrift für Soziologie der Erziehung und Sozialisation 35. Jg. (02): 155-70.

Jiow, Hee Jee, Sun Sun Lim, und Julian Lin. 2017. «Level Up! Refreshing Parental Mediation Theory for Our Digital Media Landscape: Parental Mediation of Video Gaming». Communication Theory 27 (3): 309-328. http://doi.wiley. com/10.1111/comt.12109.

Jurczyk, Karin, Andreas Lange, und Barbara Thiessen, Hrsg. 2014. Doing Family: warum Familienleben heute nicht mehr selbstverständlich ist. Weinheim Basel: Beltz Juventa.

Kammerl, Rudolf, Lena Hirschhäuser, Moritz Rosenkranz, Christiane Schwinge, Sandra Hein, Lutz Wartberg, und Kay Uwe Petersen. 2012. EXIF - Exzessive Internetnutzung in Familien: Zusammenhänge zwischen der exzessiven Computer- und Internetnutzung Jugendlicher und dem (medien)erzieherischen Handeln in den Familien. Lengerich: Pabst Science Publishers.

Kammerl, Rudolf, Jane Müller, Claudia Lampert, Marcel Rechlitz, und Katrin Potzel. 2020. «Kommunikative Figurationen - ein theoretisches Konzept zur Beschreibung von Sozialisationsprozessen und deren Wandel in mediatisierten Gesellschaften? In Bewegungen». Beiträge zum 26. Kongress der Deutschen Gesellschaft für Erziehungswissenschaft., herausgegeben von Isabell van Ackeren, Helmut Bremer, Fabian Kessl, Karl Düsseldorff, Hans-Christoph Koller, Nicolle Pfaff, Carolin Rotter., Dominique Klein und Ulrich Salaschek: 377-388. Opladen, Berlin, Toronto: Verlag Barbara Budrich.

Krotz, Friedrich. 2014. «6. Mediatization as a mover in modernity: social and cultural change in the context of media change». In Mediatization of Communication, herausgegeben von Knut Lundby. Berlin, Boston: DE GRUYTER. https:// doi.org/10.1515/9783110272215.131.

Krotz, Friedrich. 2017. «Sozialisation in mediatisierten Welten. Mediensozialisation in der Perspektive des Mediatisierungsansatzes». In Mediatisierung und Mediensozialisation: Prozesse - Räume - Praktiken, herausgegeben von Dagmar Hoffmann, Friedrich Krotz, und Wolfgang Reißmann, 21-40. Medien • Kultur - Kommunikation. Wiesbaden: Springer Fachmedien Wiesbaden. https://doi. org/10.1007/978-3-658-14937-6_2. 
Knop, Karin, Dorothée Hefner, Stefanie Schmitt, und Peter Vorderer. 2015. Mediatisierung mobil. Schriftenreihe Medienforschung der Landesanstalt für Medien Nordrhein-Westfalen. Bd. 77. Leipzig: Vistas.

Kühn, Joana, und Claudia Lampert. 2015. Mobile Internetnutzung von Kindern und Jugendlichen: eine qualitative Studie zur Smartphone- und Tablet-Nutzung von Zwei- bis 14-Jährigen. Arbeitspapiere des Hans-Bredow-Instituts 35. Hamburg: Hans-Bredow-Institut für Medienforschung.

Lampert, Claudia, Christiane Schwinge, Rudolf Kammerl, Lena Hirschhäuser, und Landesanstalt für Medien Nordrhein-Westfalen, Hrsg. 2012. Computerspiele(n) in der Familie: Computerspielesozialisation von Heranwachsenden unter besonderer Berücksichtigung genderspezifischer Aspekte. Lfm-Dokumentation 47. Düsseldorf: Landesanst. für Medien Nordrhein-Westfalen.

Livingstone, Sonia, und Leslie Haddon. 2009. EU Kids Online: Final Report. London: London school of economics and political science. http://eprints.lse.ac.uk/id/ eprint/24372.

Lüders, Jenny. 2007. Ambivalente Selbstpraktiken: eine Foucault'sche Perspektive auf Bildungsprozesse in Weblogs. Theorie bilden 8. Bielefeld: Transcript-Verl.

Mascheroni, Giovanna, und Andrea Cuman. 2014. Net Children Go Mobile: Final Report. Milano: Educatt.

Mead, George Herbert. 1968. Geist, Identität und Gesellschaft aus der Sicht des Sozialbehaviorismus. Frankfurt am Main: Suhrkamp.

Mesch, Gustavo S. 2006. «Family Relations and the Internet: Exploring a Family Boundaries Approach». Journal of Family Communication 6 (2): 119-38. https:// doi.org/10.1207/s15327698jfc0602_2.

Mikos, Lothar, Dagmar Hoffmann, und Rainer Winter, Hrsg. 2007. Mediennutzung, Identität und Identifikationen: die Sozialisationsrelevanz der Medien im Selbstfindungsprozess von Jugendlichen. Jugendforschung. Weinheim: Juventa-Verl.

Münte-Goussar, Stephan. 2016. «Medienbildung, Schulkultur und Subjektivierung». In Medien - Wissen - Bildung: Medienbildung wozu?, herausgegeben von Theo Hug, Tanja Kohn, und Petra Missomelius. Innsbruck University Press. http://library.oapen.org/handle/20.500.12657/29669.

Nathanson, Amy I. 1999. "Identifying and Explaining the Relationship Between Parental Mediation and Children's Aggression». Communication Research 26 (2): 124-43. https://doi.org/10.1177/009365099026002002.

Nathanson, Amy I. 2015. "Media and the Family: Reflections and Future Directions». Journal of Children and Media 9 (1): 133-39. https://doi.org/10.1080/1748 2798.2015.997145.

Nohl, Arnd-Michael, und Christoph Wulf, Hrsg. 2013. Mensch und Ding. Die Materialität pädagogischer Prozesse. Sonderheft 25 der Zeitschrift für Erziehungswissenschaft. Wiesbaden: Springer. 
Oevermann, Ulrich, Tilman Allert, Elisabeth Konau und Jürgen Krambeck. 1979. "Die Methodologie einer "objektiven Hermeneutik” und ihre allgemeine forschungslogische Bedeutung in den Sozialwissenschaften». In Interpretative Verfahren in den Sozial- und Textwissenschaften, herausgegeben von Hans-Georg Soeffner. 352-434. Stuttgart: Metzler.

Paus-Haase, Ingrid, Stefan Aufenanger, und Uwe Mattusch. 2000. Hörfunknutzung von Kindern: Bestandsaufnahme und Entwicklungschancen des Kinderhörfunks im dualen System: eine Studie. Schriftenreihe der NLM, Bd. 7. Berlin: Vistas.

Paus-Haase, Ingrid, und Bernd Schorb. 2000. Qualitative Kinder- und Jugendmedienforschung: Theorie und Methoden; ein Arbeitsbuch. München: kopaed.

Paus-Hasebrink, Ingrid, Klaus Neumann-Braun, Uwe Hasebrink, und Stefan Aufenanger, Hrsg. 2004. Medienkindheit - Markenkindheit: Untersuchungen zur multimedialen Verwertung von Markenzeichen für Kinder. Bd. 18. Schriftenreihe der LPR Hessen. München: kopaed.

Paus-Hasebrink, Ingrid, und Jasmin Kulterer. 2014. Praxeologische Mediensozialisationsforschung. Nomos. https://doi.org/10.5771/9783845252827.

Paus-Hasebrink, Ingrid, und Uwe Hasebrink. 2014. «Kommunikative Praxen im Wandel». In MedienJournal, 38(1), 4-14. https://doi.org/10.24989/medienjournal.v38i1.106.

Paus-Hasebrink, Ingrid, Hrsg. 2017. Langzeitstudie zur Rolle von Medien in der Sozialisation sozial benachteiligter Heranwachsender. Lebensphase Jugend. Lebensweltbezogene Medienforschung: Angebote - Rezeption - Sozialisation Band 5. Baden-Baden: Nomos.

Paus-Hasebrink, Ingrid, Philip Sinner, Jasmin Kulterer, und Andreas Oberlinner. 2017. «Methodologische und methodische Herausforderungen: Zum Design der Langzeit-Panelstudie». In Langzeitstudie zur Rolle von Medien in der Sozialisation sozial benachteiligter Heranwachsender. Lebensphase Jugend. Lebensweltbezogene Medienforschung: Angebote - Rezeption - Sozialisation, herausgegeben von Ingrid Paus-Hasebrink. Band 5: 45-68. Baden-Baden: Nomos.

Paus-Hasebrink, Ingrid, Jasmin Kulterer, und Philip Sinner. 2019. Social Inequality, Childhood and the Media: A Longitudinal Study of the Mediatization of Socialisation. Cham: Springer International Publishing. https://doi.org/10.1007/9783-030-02653-0.

Pfetsch, Jan. 2018. «Jugendliche Nutzung digitaler Medien und elterliche Medienerziehung - Ein Forschungsüberblick». Praxis der Kinderpsychologie und Kinderpsychiatrie 67 (2): 110-33. https://doi.org/10.13109/prkk.2018.67.2.110.

Schaan, Violetta K., und André Melzer. 2015. «Parental Mediation of Children's Television and Video Game Use in Germany: Active and Embedded in Family Processes». Journal of Children and Media 9 (1): 58-76. https://doi.org/10.1080/1 7482798.2015.997108.

Schlör, Katrin. 2016. Medienkulturen in Familien in belasteten Lebenslagen: eine Langzeitstudie zu medienbezogenem Doing Family als Bewältigungsressource. Reihe medienpädagogische Praxisforschung, Band 1l. München: kopaed. 
Schorb, Bernd, und Helga Theunert. 2000. «Kontextuelles Verstehen der Medienaneignung». In Qualitative Kinder- und Jugendmedienforschung: Theorie und Methoden; ein Arbeitsbuch, herausgegeben von Ingrid Paus-Haase und Bernd Schorb, 33-57. München: KoPäd-Verl.

Schuegraf, Martina. 2008. Medienkonvergenz und Subjektbildung: mediale Interaktionen am Beispiel von Musikfernsehen und Internet. 1. Aufl. Medienbildung und Gesellschaft, Bd. 5. Wiesbaden: VS Verlag für Sozialwissenschaften. https:// doi.org/10.1007/978-3-531-90805-2.

Schuegraf, Martina. 2010. «Medienkonvergenz». In Handbuch Mediensozialisation, herausgegeben von Ralf Vollbrecht und Claudia Wegener, 1. Aufl, 287-98. Wiesbaden: VS Verlag für Sozialwissenschaften.

Schütz, Alfred. 1974. Der sinnhafte Aufbau der sozialen Welt: eine Einleitung in die verstehende Soziologie. Zweite Auflage, 9.-10. Tausend. Suhrkamp Taschenbücher Wissenschaft 92. Frankfurt am Main: Suhrkamp.

Six, Ulrike, Roland Gimmler, und Ines Vogel. 2002. Medienerziehung in der Familie: Hintergrundinformationen und Anregungen für medienpädagogische Elternarbeit. Themen, Thesen, Theorien 20. Kiel: ULR.

Spanhel, Dieter. 2006. Medienerziehung: Erziehungs- und Bildungsaufgaben in der Mediengesellschaft. Handbuch Medienpädagogik 03. Stuttgart: Klett-Cotta.

Steinberg, Laurence. 2001. "We Know Some Things: Parent-Adolescent Relationships in Retrospect and Prospect». Journal of Research on Adolescence 11 (1): 1-19. https://doi.org/10.1111/1532-7795.00001.

Subrahmanyam, Kaveri, und Patricia Greenfield. 2008. «Online Communication and Adolescent Relationships》. The Future of Children 18: 119-46. https://futureofchildren.princeton.edu/sites/futureofchildren/files/media/children_and_ electronic_media_18_01_fulljournal.pdf.

Süss, Daniel. 2004. Mediensozialisation von Heranwachsenden: Dimensionen, Konstanten, Wandel. 1. Auflage. Wiesbaden: VS, Verlag für Sozialwissenschaften. https://doi.org/10.1007/978-3-531-90045-2.

Süss, Daniel, Claudia Lampert, und Christine W. Wijnen. 2018. Medienpädagogik. Ein Studienbuch zur Einführung. 3., überarbeitete und aktualisierte Aufl. Wiesbaden: Springer Fachmedien Wiesbaden. https://doi.org/10.1007/978-3-65819824-4.

Theunert, Helga, und Christa Gebel. 2000. Lehrstücke fürs Leben in Fortsetzung: Serienrezeption zwischen Kindheit und Jugend. BLM-Schriftenreihe 63. München: Fischer.

Theunert, Helga, und Andreas Lange. 2012. «Doing Family im Zeitalter von Mediatisierung und Pluralisierung». merz. Medien + Erziehung 56, Familie und Medien (2012/02): 10-20.

Tillmann, Angela. 2008. Identitätsspielraum Internet: Lernprozesse und Selbstbildungspraktiken von Mädchen und jungen Frauen in der virtuellen Welt. Geschlechterforschung. Weinheim: Juventa. 
Tillmann, Angela, und Kai-Uwe Hugger. 2014. "Mediatisierte Kindheit - Aufwachsen in mediatisierten Lebenswelten». In Handbuch Kinder und Medien, herausgegeben von Angela Tillmann, Sandra Fleischer und Kai-Uwe Hugger. Wiesbaden: Springer VS: 31-45. https://doi.org/10.1007/978-3-531-18997-0_2.

Treibel, Annette. 2009. «Figurations- und Prozesstheorie». In Handbuch Soziologische Theorien, herausgegeben von Georg Kneer und Markus Schroer, 133-60. Wiesbaden: VS Verlag für Sozialwissenschaften. https://doi.org/10.1007/9783-531-91600-2_7.

Vaala, Sarah E., und Amy Bleakley. 2015. «Monitoring, Mediating, and Modeling: Parental Influence on Adolescent Computer and Internet Use in the United States». Journal of Children and Media 9 (1): 40-57. https://doi.org/10.1080/1748 2798.2015.997103.

Valkenburg, Patti M., Marina Krcmar, Allerd L. Peeters, und Nies M. Marseille. 1999. "Developing a Scale to Assess Three Styles of Television Mediation: "Instructive Mediation," "Restrictive Mediation," and "Social Coviewing"». Journal of Broadcasting \& Electronic Media 43 (1): 52-66. https://doi. org/10.1080/08838159909364474.

Valkenburg, Patti M., Jessica Taylor Piotrowski, Jo Hermanns, und Rebecca de Leeuw. 2013. «Developing and Validating the Perceived Parental Media Mediation Scale: A Self-Determination Perspective: Parental Mediation Scale». Human Communication Research 39 (4): 445-69. https://doi.org/10.1111/hcre.12010.

Vollbrecht, Ralf. 2003. "Aufwachsen in Medienwelten». In Mediensozialisation: pädagogische Perspektiven des Aufwachsens in Medienwelten, herausgegeben von Karsten Fritz, Stephan Sting, und Ralf Vollbrecht, 13-24. Opladen: Leske + Budrich. https://doi.org/10.1007/978-3-322-87371-2_2.

Wagner, Ulrike, Christa Gebel, und Claudia Lampert. 2013. Zwischen Anspruch und Alltagsbewältigung: Medienerziehung in der Familie. Schriftenreihe Medienforschung der Landesanstalt für Medien Nordrhein-Westfalen 72. Berlin: Vistas-Verl. [u.a.]. https://www.medienanstalt-nrw.de/fileadmin/user_upload/ lfm-nrw/Foerderung/Forschung/Dateien_Forschung/LfM-Band-72.pdf.

Wagner, Ulrike, und Claudia Lampert. 2013. «Zur Notwendigkeit einer ressourcenorientierten Mediensozialisationsforschung». In Medienwelten im Wandel, herausgegeben von Christine W. Wijnen, Sascha Trültzsch, und Christina Ortner, 223-36. Wiesbaden: Springer Fachmedien Wiesbaden. https://doi. org/10.1007/978-3-531-19049-5_16.

Wagner, Ulrike, und Helga Theunert. 2006. Neue Wege durch die konvergente Medienwelt: Studie im Auftrag der Bayerischen Landeszentrale für neue Medien (BLM). BLM-Schriftenreihe 85. München: Fischer.

Wartberg, Lutz, Levente Kriston, Michaela Kramer, Anja Schwedler, Tanja M. Lincoln, und Rudolf Kammerl. 2017. "Internet Gaming Disorder in Early Adolescence: Associations with Parental and Adolescent Mental Health». European Psychiatry 43 (Juni): 14-18. https://doi.org/10.1016/j.eurpsy.2016.12.013.

Wegener, Claudia. 2008. Medien, Aneignung und Identität: "Stars" im Alltag jugendlicher Fans. 1. Aufl. Wiesbaden: VS, Verl. für Sozialwiss. https://doi. org/10.1007/978-3-531-90833-5. 
Wegener, Claudia. 2016. Aufwachsen Mit Medien. Medienwissen Kompakt. VS Verlag für Sozialwissenschaften. https://doi.org/10.1007/978-3-658-00843-7.

West, Candace, und Don H. Zimmerman. 1987. «Doing Gender». Gender \& SocietY I (2): 125-51. https://doi.org/10.1177/0891243287001002002.

Wieler, Petra. 2010. «Buch - Lesesozialisation». In Handbuch Mediensozialisation, herausgegeben von Ralf Vollbrecht und Claudia Wegener, 1. Aufl, 231-40. Wiesbaden: VS Verlag für Sozialwissenschaften.

Wiesemann, Jutta, und Inka Fürtig. 2018. «Kindheit zwischen Smartphone und pädagogischem Schulalltag». In Institutionalisierungen von Kindheit. Childhood studies zwischen Soziologie und Erziehungswissenschaft, herausgegeben von Tanja Betz, Reihe Kindheiten - Neue Folge, 195-212. Weinheim/München: Beltz Juventa.

Wolf, Karsten D., und Konstanze Wegmann. 2020. "Situationsanalyse in Der medienpädagogischen Mediatisierungsforschung: Kommunikative Figurationen Des Informellen Lernens». MedienPädagogik: Zeitschrift für Theorie und Praxis der Medienbildung 15 (Jahrbuch Medienpädagogik):79-119. https://doi. org $/ 10.21240 / \mathrm{mpaed} / \mathrm{jb15} / 2020.03 .05 . X$

Wrana, Daniel, Marion Ott, Kerstin Jergus, Antje Langer, und Sandra Koch. 2014. «Diskursforschung in der Erziehungswissenschaft». In Diskursforschung: ein interdisziplinäres Handbuch, herausgegeben von Johannes Angermuller, Martin Nonhoff und Johannes Angermüller, 224-38. DiskursNetz, Band 1. Bielefeld: Transcript. https://doi.org/10.14361/transcript.9783839427224.224. 